\title{
ON THE ZEROS OF SUCCESSIVE DERIVATIVES OF INTEGRAL FUNCTIONS*
}

\author{
BY \\ I. J. SCHOENBERG \\ INTRODUCTION
}

1. Let $\eta_{0}, \eta_{1}, \eta_{2}, \ldots$ be a sequence of complex numbers and $f(x)$ an analytic function. The expansion in series of polynomials

$$
f(x)=\sum_{n=0}^{\infty} f^{(n)}\left(\eta_{n}\right) p_{n}(x)
$$

where

$$
p_{n}(x)=\int_{\eta_{0}}^{x} d x_{1} \int_{\eta_{1}}^{x_{1}} d x_{2} \cdots \int_{\eta_{n-1}}^{x_{n-1}} d x_{n} \quad\left(n \geqq 1, p_{0}(x)=1\right),
$$

was made the subject of important recent investigations by W. Goncharoff $\dagger$ and J. M. Whittaker. $\ddagger$ Goncharoff calls (1) a generalized Abel series; it is readily found to be valid if $f(x)$ is a polynomial and reduces to the Taylor expansion of $f(x)$ about the point $x=a$ if $\eta_{0}=\eta_{1}=\cdots=a$. Whittaker investigated the expansion (1) in the case when every $\eta_{n}=1$ or $-1 \S$ and proved the following theorem:

Let $f(x)$ be an integral function for which

$$
\varlimsup_{r \rightarrow \infty} \frac{\log M(r)}{r}=\gamma<\frac{1}{2},
$$

$M(r)$ denoting the maximum modulus of the function on the circle $|x|=r$. Let $\left\{\eta_{n}\right\}$ be a sequence with $\eta_{n}= \pm 1$. Then the expansion (1) is valid in the circle

* Presented to the Society, January 2, 1936; received by the editors November 19, 1935.

$\dagger$ W. Goncharoff, Recherches sur les dérivées successives des fonctions analytiques. Généralisation de la série d'Abel, Annales Scientifiques de l'École Normale, vol. 47 (1930), pp. 1-78.

$\ddagger \mathrm{J}$. M. Whittaker, On Lidstone's series and two-point expansions of analytic functions, Proceedings of the London Mathematical Society, (2), vol. 36 (1933-34), pp. 451-469.

$\S$ Whittaker actually considers the case $\eta_{n}=0$ or 1 , which may be reduced to the case $\eta_{n}= \pm 1$ by an obvious linear transformation of the $x$-plane.

|| Loc. cit., Theorem 7, p. 468. A weaker form of this theorem, the number $1 / 2$ on the right side of (3) being replaced by $1 /(2 e)$, is a particular case of a general theorem proved by Goncharoff, loc. cit., pp. 35-36, and by S. Takenaga, Proceedings of the Physico-Mathematical Society of Japan, vol. 13 (1931), pp. 111-132. 


$$
|x|<\frac{1}{\gamma}-1 \text {. }
$$

An immediate corollary stated by Whittaker is as follows.

If $f(x)$ is an integral function satisfying the condition (3) and

$$
f^{(n)}(-1) f^{(n)}(1)=0 \quad(n=0,1,2, \cdots),
$$

then $f(x)=0$ identically.

2. Whittaker remarks, by consideration of the function $\sin (x+1) \pi / 4$, of type $\gamma=\pi / 4$, which satisfies (5) without vanishing identically, that the number $\frac{1}{2}$, on the right side of (3), can not be replaced by any number larger than $\pi / 4$. He conjectures that $\pi / 4$ is the best constant in the condition (3). A verification of this conjecture is contained in the following

THEOREM 1. Let $f(x)$ be an integral function for which

$$
\varlimsup_{r \rightarrow \infty} \frac{\log M(r)}{r}<\frac{\pi}{4} \text {. }
$$

Let $\left\{\eta_{n}\right\}$ be a sequence of real numbers, with $-1 \leqq \eta_{n} \leqq 1$. Then the expansion (1) is valid throughout the complex plane, absolutely and uniformly in every finite region.

As above we have the following

COROLLARY. If an integral function $f(x)$, satisfying (6), is such that every one of its derivatives $f(x), f^{\prime}(x), f^{\prime \prime}(x), \cdots$ vanishes somewhere between -1 and +1 , then $f(x)=0$ identically.

It might be of interest to point out the following consequence which is obtained by transforming these results by means of $z=(x+1) / 4$ :

A mong all integral functions $f(z) \not \equiv 0$ which are such that $f(z), f^{\prime}(z), f^{\prime \prime}(z), \ldots$ vanish somewhere within the interval $0 \leqq z \leqq \frac{1}{2}$, the function $\sin \pi z$ is the one of least growth.

3. To establish Theorem 1 we shall use essentially the same tools with which Whittaker proved his results stated above. However, these did not suffice entirely in the present problem for the following reason. Whittaker could solve completely by his method the questions regarding the validity of Lidstone's series (loc. cit., Theorem 2, p. 455) and of (1) in the case when $\eta_{n}=(-1)^{n}$ (loc. cit., $\S 6$, p. 458 ), because the polynomials of both these expansions reduce essentially to Appell sequences, and could thus be conveniently estimated by means of their generating functions. Our polynomials (1) $\left(-1 \leqq \eta_{\nu} \leqq 1\right)$ do not form an Appell sequence, for, in the successive integra- 
tions in (2), the lower limits of integration proceed in the wrong direction, so to speak. This difficulty is overcome by the consideration of the polynomials (21) which do form an Appell sequence. The solution of an extremal problem (Lemma 1) leads to an estimate for the polynomials (21) (Lemma 2) which holds uniformly in the $\eta$ 's within $(-1,1)$; then surely this result may be applied to the polynomials (2) as well, provided $-1 \leqq \eta_{\nu} \leqq 1$.

I. AN EXTREMAL PROBLEM CONCERNING THE POLYNOMIALS $p_{n}(x)$

4. An important step in the proof of Theorem 1 is the solution of the following extremal problem: Let $p_{n}(x)$ be a real polynomial of degree $n(>0)$ of the form $p_{n}(x)=x^{n} / n !+\cdots$. Let $p_{n}(x), p_{n}^{\prime}(x), \cdots, p_{n}{ }^{(n-1)}(x)$ all have at least one zero within the interval $-1 \leqq x \leqq 1$. What is the largest value of $\left|p_{n}(0)\right|$ ?

This problem is solved by the following

Lemma 1. The largest possible value of $\left|p_{n}(0)\right|$ is $\left|P_{n}(0)\right|$, where

$$
P_{n}(x)=\int_{-1}^{x} d x_{1} \int_{+1}^{x_{1}} d x_{2} \cdots \int_{(-1)^{n}}^{x_{n-1}} d x_{n}
$$

As

$$
p_{n}(x)=\int_{\eta_{0}}^{x} d x_{1} \int_{\eta_{1}}^{x_{1}} d x_{2} \cdots \int_{\eta_{n-1}}^{x_{n-1}} d x_{n} \quad\left(-1 \leqq \eta_{\nu} \leqq 1\right)
$$

is the general type of polynomials considered in our extremal problem, Lemma 1 is equivalent with the following inequality

$$
\begin{array}{r}
\left|\int_{-1}^{0} d x_{1} \int_{+1}^{x_{1}} d x_{2} \cdots \int_{(-1)^{n}}^{x_{n-1}} d x_{n}\right| \geqq\left|\int_{\eta_{0}}^{0} d x_{1} \int_{\eta_{1}}^{x_{1}} d x_{2} \cdots \int_{\eta_{n-1}}^{x_{n-1}} d x_{n}\right|, \\
\text { if }-1 \leqq \eta_{\nu} \leqq 1 .
\end{array}
$$

We shall find it more convenient, however, to prove the more inclusive inequality

$$
\left|\int_{-1}^{x} d x_{1} \int_{+1}^{x_{1}} d x_{2} \cdots \int_{(-1)^{n}}^{x_{n}-1} d x_{n}\right| \geqq\left|\int_{\eta_{0}}^{x} d x_{1} \int_{\eta_{1}}^{x_{1}} d x_{2} \cdots \int_{\eta_{n-1}}^{x_{n-1}} d x_{n}\right|,
$$

$$
\text { if } 0 \leqq x \leqq 1,-1 \leqq \eta_{\nu} \leqq 1 \text {. }
$$

We shall therefore prove that for any fixed $x$, with $0 \leqq x \leqq 1,\left|P_{n}(x)\right|$ is the largest possible value of $\left|p_{n}(x)\right| .^{*}$

Let

- This implies, for reasons of symmetry, that, if $-1 \leqq x \leqq 0$, the largest possible value of $\left|p_{n}(x)\right|$ is $\left|P_{n}(-x)\right|$. The answer to our extremal problem is an entirely different one if $x$ has a fixed value, with $\left|x_{1}\right|>1$, and is of no interest in the present investigation. 


$$
\begin{aligned}
\Phi_{n}(x ; \eta) & =\Phi_{n}\left(x ; \eta_{0}, \eta_{1}, \ldots, \eta_{n-1}\right) \\
& =\left|\int_{\eta_{0}}^{x} d x_{1}\right| \int_{\eta_{1}}^{x_{1}} d x_{2} \ldots\left|\int_{\eta_{n-1}}^{x_{n-1}} d x_{n}\right| \ldots \mid .
\end{aligned}
$$

From (7), (8), and (11) we get

$$
\Phi_{n}(x ; \eta) \geqq\left|p_{n}(x)\right|, \quad \Phi_{n}\left(x ;-1, \cdots,(-1)^{n}\right)=\left|P_{n}(x)\right| ;
$$

hence (10) will be proved if we can show that

$$
\begin{aligned}
\Phi_{n}\left(x ;-1, \cdots,(-1)^{n}\right) \geqq \Phi_{n}\left(x ; \eta_{0}, \cdots, \eta_{n-1}\right), & \\
& \text { for } 0 \leqq x \leqq 1,-1 \leqq \eta_{\nu} \leqq 1 .
\end{aligned}
$$

Let $x(0 \leqq x \leqq 1)$ be kept fixed; $\Phi_{n}(x ; \eta)$ is a continuous function of $\left(\eta_{0}, \cdots, \eta_{n-1}\right)$ and (12) states that it reaches its maximum value within the cube $-1 \leqq \eta_{\nu} \leqq 1$ at a certain corner, namely, $(\eta)=\left(-1, \cdots,(-1)^{n}\right)$. We shall prove now that the maximum value within the cube is necessarily attained at some one of the $2^{n}$ corners $\left(\eta_{\nu}= \pm 1\right)$ of the cube. For let $\left(\eta_{0}, \eta_{1}, \cdots, \eta_{n-1}\right)$ be a point at which the maximum within the cube is reached. From (11) it is obvious that $-1<\eta_{0}<1$ is impossible, for $\Phi_{n}(x ; \eta)$ can be further increased by increasing the difference $\left|x-\eta_{0}\right|$. Hence $\eta_{0}= \pm 1$. Let

$$
\eta_{\nu}= \pm 1 \quad(\nu=0,1, \cdots, k-1), \quad-1<\eta_{k}<1,
$$

where $k$ may take any value from 1 to $n-1$. In view of (13) we can write (14) $\Phi_{n}(x ; \eta)=\int \ddot{\mathscr{D}} \int\left|\int_{\eta_{k}}^{x_{k}} \Phi_{n-k-1}\left(x_{k+1} ; \eta_{k+1}, \cdots, \eta_{n-1}\right) d x_{k+1}\right| d x_{1} \cdots d x_{k}$, where the integrand $\left|\int_{\eta_{k}}^{x_{k}} \Phi_{n-k-1} d x_{k+1}\right|$ is integrated in the ordinary sense over a certain polyhedral domain $\mathcal{D}$, in the space of the variables $\left(x_{1}, \cdots, x_{k}\right)$, which is entirely contained within the cube $-1 \leqq x_{\nu} \leqq 1(\nu=1,2, \cdots, k){ }^{*}$ In equation (14) we now keep everything fixed but $\eta_{k}$, which may vary from -1 to +1 . In view of the formula

$$
\begin{aligned}
& \frac{\partial}{\partial \eta_{k}}\left|\int_{\eta_{k}}^{x_{k}} \Phi_{n-k-1}\left(x_{k+1} ; \eta_{k+1}, \cdots, \eta_{n-1}\right) d x_{k+1}\right| \\
& \quad=\Phi_{n-k-1}\left(\eta_{k} ; \eta_{k+1}, \cdots, \eta_{n-1}\right) \operatorname{sgn}\left(\eta_{k}-x_{k}\right),
\end{aligned}
$$

(14) gives

* Thus if $k=3, \eta_{0}=-1, \eta_{1}=-1, \eta_{2}=+1$, then

$$
\Phi_{n}(x ; \eta)=\int_{-1}^{x} d x_{1} \int_{-1}^{x_{1}} d x_{2} \int_{x_{2}}^{1}\left|\int_{\eta_{3}}^{x_{3}} \Phi_{n-4} d x_{4}\right| d x_{3}
$$

and the domain $\mathcal{D}$ is defined by the inequalities

$$
-1 \leqq x_{1} \leqq x, \quad-1 \leqq x_{2} \leqq x_{1}, \quad x_{2} \leqq x_{3} \leqq 1 .
$$




$$
\begin{aligned}
& \frac{\partial}{\partial \eta_{k}} \Phi_{n}(x ; \eta) \\
& \quad \therefore \Phi_{n-k-1}\left(\eta_{k} ; \eta_{k+1}, \cdots, \eta_{n-1}\right) \int \ddot{\mathscr{D}} \int \operatorname{sgn}\left(\eta_{k}-x_{k}\right) d x_{1} \cdots d x_{k} .
\end{aligned}
$$

The integral on the right side of (15) is a non-decreasing continuous function of $\eta_{k}$ which has the value ( - volume $\mathscr{D}$ ) for $\eta_{k}=-1$ and (+ volume $\mathscr{D}$ ) for $\eta_{k}=1$; moreover this non-decreasing function may have only one interval of constancy at one end of the interval $(-1,1)$ and the function can therefore vanish at one point only, say $\eta_{k}=\xi$. The first factor $\Phi_{n-k-1}\left(\eta_{k} ; \cdots\right)$ on the right side of (15) is a continuous and positive function of $\eta_{k}$ which vanishes only for $\eta_{k}=\eta_{k+1}$. Hence $\partial \Phi_{n} / \partial \eta_{k}$, as given by (15), vanishes for $\eta_{k}=\xi$, $\eta_{k}=\eta_{k+1}$; for any other value of $\eta_{k}$, however, it is $<0$ or $>0$, according as $\eta_{k}<\xi$ or $\eta_{k}>\xi$. Hence, as $\eta_{k}$ varies from -1 to $+1, \Phi_{n}(x ; \eta)$ will decrease to a certain minimum value to increase thereafter for the remainder of this interval. This shows that our assumption (13), namely, $-1<\eta_{k}<1$, is impossible. The point where $\Phi_{n}(x ; \eta)$ reaches its maximum must therefore be found among the $2^{n}$ corners of the cube $-1 \leqq \eta_{\nu} \leqq 1$.

5. It suffices therefore to prove the inequality (12) under the restricted assumptions that all $\eta_{\nu}= \pm 1$ (and $0 \leqq x \leqq 1$, as throughout our discussion). Now all but one set of the absolute value signs in (11) may be dropped and we can write

$$
\Phi_{n}(x ; \eta)=\left|\int_{\eta_{0}}^{x} d x_{1} \int_{\eta_{1}}^{x_{1}} d x_{2} \cdots \int_{\eta_{n-1}}^{x_{n-1}} d x_{n}\right| \quad\left(\eta_{\nu}= \pm 1\right) .
$$

Since

$$
\begin{aligned}
\Phi_{n}\left(x ; 1, \eta_{1}, \cdots, \eta_{n-1}\right) & =\Phi_{n}\left(-x ;-1,-\eta_{1}, \cdots,-\eta_{n-1}\right) \\
& \leqq \Phi_{n}\left(x ;-1,-\eta_{1}, \cdots,-\eta_{n-1}\right),
\end{aligned}
$$

it suffices to consider the case $\eta_{0}=-1$ only. For $n=1$ (12) is true. We assume now (12) to hold (for $\eta_{\nu}= \pm 1$ ) if $n$ is replaced by any number $m<n$. We shall prove the inequalities

$$
\begin{array}{r}
\Phi_{n}\left(x ;-1,+1, \cdots,(-1)^{n}\right) \geqq \Phi_{n}\left(x ;-1,+1, \eta_{2}, \cdots,\right. \\
\left.\eta_{n-1}\right), \\
\left(\eta_{\nu}= \pm 1\right), \\
\Phi_{n}\left(x ;-1,+1, \cdots,(-1)^{n}\right) \geqq \Phi_{n}\left(x ;-1,-1, \eta_{2}, \cdots,\right. \\
\left.\eta_{n-1}\right), \\
\left(\eta_{v}= \pm 1\right),
\end{array}
$$

which cover all remaining possibilities.

To prove (16) we write 


$$
\begin{aligned}
\Phi_{n}\left(x ;-1, \cdots,(-1)^{n}\right)= & \int_{-1}^{0} \Phi_{n-1}\left(x_{1} ; 1,-1, \cdots\right) d x_{1} \\
& +\int_{0}^{x} \Phi_{n-1}\left(x_{1} ; 1,-1, \cdots\right) d x_{1},
\end{aligned}
$$

or

$$
\begin{aligned}
\Phi_{n}\left(x ;-1, \cdots,(-1)^{n}\right)= & \int_{-1}^{0} \Phi_{n-1}\left(-x_{1} ;-1,+1, \cdots\right) d x_{1} \\
& +\int_{0}^{x} d x_{1} \int_{x_{1}}^{1} \Phi_{n-2}\left(x_{2} ;-1,+1, \cdots\right) d x_{2},
\end{aligned}
$$

and correspondingly

$$
\begin{aligned}
\Phi_{n}\left(x ;-1,+1, \eta_{2}, \cdots, \eta_{n-1}\right)= & \int_{-1}^{0} \Phi_{n-1}\left(-x_{1} ;-1,-\eta_{2}, \cdots\right) d x_{1} \\
& +\int_{0}^{x} \cdot d x_{1} \int_{x_{1}}^{1} \Phi_{n-2}\left(x_{2} ; \eta_{2}, \cdots, \eta_{n-1}\right) d x_{2} .
\end{aligned}
$$

In the first integrals in (18) and (19) we have $0 \leqq-x_{1} \leqq 1$, and therefore the integrand in (18) is $\geqq$ the integrand in (19), on account of our assumption. In the second integrals in (18) and (19) we have $0 \leqq x_{2} \leqq 1$ and for a similar reason the proof of (16) is completed.

In order to prove (17) we consider the function

$$
g(x)=\Phi_{n}\left(x ;-1,+1, \cdots,(-1)^{n}\right)-\Phi_{n}\left(x ;-1,-1, \eta_{2}, \cdots, \eta_{n-1}\right)
$$

and we have to prove that

$$
g(x) \geqq 0 \quad \text { if } \quad 0 \leqq x \leqq 1 .
$$

This will be done in three steps. We show first that $g^{\prime}(x)$ is a non-increasing function. Indeed

$$
g(x)=\int_{-1}^{x} d x_{1} \int_{x_{1}}^{1} \Phi_{n-2}\left(x_{2} ;-1, \cdots\right) d x_{2}-\int_{-1}^{x} d x_{1} \int_{-1}^{x_{1}} \Phi_{n-2}\left(x_{2} ; \eta_{2}, \cdots\right) d x_{2},
$$

whence

$g^{\prime}(x)=\int_{x}^{1} \Phi_{n-2}\left(x_{2} ;-1, \cdots\right) d x_{2}-\int_{-1}^{x} \Phi_{n-2}\left(x_{2} ; \eta_{2}, \cdots\right) d x_{2}$,

which proves the statement, for $g^{\prime}(x)$ is a sum of two non-increasing functions. In view of this fact, (20) will be established if we can show that $g(0) \geqq 0$ and $g(1) \geqq 0$, i.e., that (17) holds for $x=0$ and $x=1$. To prove (17) for $x=0$ we notice that 


$$
\begin{aligned}
\Phi_{n}\left(0 ;-1,1, \cdots,(-1)^{n}\right) & =\int_{-1}^{0} \Phi_{n-1}\left(x_{1} ; 1,-1, \cdots\right) d x_{1} \\
& =\int_{-1}^{0} \Phi_{n-1}\left(-x_{1} ;-1,+1,-1, \cdots\right) d x_{1}
\end{aligned}
$$

similarly

$$
\Phi_{n}\left(0 ;-1,-1, \eta_{2}, \cdots\right)=\int_{-1}^{0} \Phi_{n-1}\left(-x_{1} ; 1,-\eta_{2}, \cdots\right) d x_{1},
$$

and the integrand of the second integral is superior $(\geqq)$ to the integrand of the third integral since $0 \leqq-x_{1} \leqq 1$. To prove (17) for $x=1$ we notice that

$$
\begin{aligned}
\Phi_{n}\left(1 ;-1,-1, \eta_{2}, \cdots\right) & =\int_{-1}^{1} \Phi_{n-1}\left(x_{1} ;-1, \eta_{2}, \cdots\right) d x_{1} \\
& =\int_{-1}^{1} \Phi_{n-1}\left(-x_{1} ; 1,-\eta_{2}, \cdots\right) d x_{1} \\
& =\int_{-1}^{1} \Phi_{n-1}\left(x_{1} ; 1,-\eta_{2}, \cdots\right) d x_{1} \\
& =\Phi_{n}\left(1 ;-1,1,-\eta_{2}, \cdots\right) \\
& \leqq \Phi_{n}\left(1 ;-1,1,-1, \cdots,(-1)^{n}\right)
\end{aligned}
$$

the last inequality resulting from (16) for $x=1$. This completes the proof of Lemma 1.

II. AN ESTIMATE OF $p_{n}(x)$ OF LOWEST ORDER IN $n$ FOR $n \rightarrow \infty$

6 . Instead of the polynomials $p_{n}(x)$ defined by (2) we shall now investigate the sequence of polynomials

$$
q_{n}(x)=\int_{\eta_{n-1}}^{x} d x_{1} \int_{\eta_{n-2}}^{x_{1}} d x_{2} \cdots \int_{\eta_{0}}^{x_{n-1}} d x_{n} \quad\left(-1 \leqq \eta_{\nu} \leqq 1 ; q_{0}(x)=1\right) .
$$

This is a sequence of Appell polynomials, i.e., satisfying the recurrence relation $q_{n}^{\prime}(x)=q_{n-1}(x)$. It is known and easily shown* that the $q_{n}(x)$ have a generating function of the type

$$
e^{x t} f(t)=\sum_{n=0}^{\infty} q_{n}(x) t^{n}
$$

where 144.

* P. Appell, Sur une classe de polynômes, Annales de l'École Normale, (2), vol. 9 (1880), pp. 119- 


$$
f(t)=1+\frac{a_{1}}{1 !} t+\frac{a_{2}}{2 !} t^{2}+\cdots .
$$

This series, if multiplied formally with the power series expansion of $e^{x t}$, gives

$$
q_{n}(x)=\frac{1}{n !}\left\{x^{n}+\left(\begin{array}{l}
n \\
1
\end{array}\right) a_{1} x^{n-1}+\cdots+a_{n}\right\} .
$$

A particular sequence of polynomials of the form (21) will be of special interest, namely,

$$
r_{n}(x)=\int_{(-1)^{n}}^{x} d x_{1} \int_{(-1)^{n-1}}^{x_{1}} d x_{2} \cdots \int_{-1}^{x_{n-1}} d x_{n} \quad\left(r_{0}(x)=1\right) .
$$

To find the generating function

$$
e^{x t} g(t)=\sum_{n=0}^{\infty} r_{n}(x) t^{n}
$$

of the polynomials $r_{n}(x)$ we note that they are characterized among all Appell sequences by the conditions

$$
r_{n}(+1)=0 \quad(n=2,4,6, \cdots), \quad r_{n}(-1)=0 \quad(n=1,3,5, \cdots) ;
$$

these conditions are equivalent to the requirement that $e^{t} g(t)-1$ be an odd function of $t$ and $e^{-t} g(t)$ be an even function, i.e.,

$$
e^{t} g(t)-1=-e^{-t} g(-t)+1, \quad e^{-t} g(t)=e^{t} g(-t),
$$

whence we get

$$
g(t)=e^{t} \operatorname{sech} 2 t .
$$

Now (26) becomes

$$
e^{(x+1) t} \operatorname{sech} 2 t=\sum_{n=0}^{\infty} r_{n}(x) t^{n} .
$$

7. A convenient expression for $r_{n}(x)$ can now be found by a customary method. Let

$$
r_{n}(x ; \rho)=\frac{1}{2 \pi i} \int_{|t|=\rho} t^{-n-1} e^{(x+1) t} \operatorname{sech} 2 t d t,
$$

where the integration is taken in the positive sense of rotation along the circumference $|t|=\rho$.

Noting that $t= \pm \pi i / 4$ are the only (simple) poles of sech $2 t$ within the circle $|t| \leqq 1$, we find by Cauchy's theorem of residues 


$$
r_{n}(x ; 1)-r_{n}\left(x ; \frac{\pi}{8}\right)=\frac{(-1)^{[n / 2]+1}}{2}\left(\frac{4}{\pi}\right)^{n+1}\left\{\begin{array}{l}
\cos \\
\sin
\end{array}\right\} \frac{\pi}{4}(x+1),\left\{\begin{array}{l}
n \text { even } \\
n \text { odd }
\end{array}\right\},
$$

where on the right side we have the factor $\cos (x+1) \pi / 4$ or $\sin (x+1) \pi / 4$, according as $n$ is even or odd. In view of (27) we have

$$
r_{n}\left(x ; \frac{\pi}{8}\right)=r_{n}(x)
$$

and finally

$$
r_{n}(x)=\frac{\left(-1^{[n / 2]}\right)}{2}\left(\frac{4}{\pi}\right)^{n+1}\left\{\begin{array}{l}
\cos \\
\sin
\end{array}\right\} \frac{\pi}{4}(x+1)+r_{n}(x ; 1), \quad\left\{\begin{array}{l}
n \text { even } \\
n \text { odd }
\end{array}\right\}
$$

where

$$
\left|r_{n}(x ; 1)\right| \leqq(2 \pi)^{-1} e^{|x|} \int_{|t|=1}\left|e^{t} \operatorname{sech} 2 t\right| \cdot|d t| .
$$

Formulas (29) and (30) reveal some interesting properties of these polynomials, as, for example, the relations

(31) $\lim _{n \rightarrow \infty} \frac{r_{2 n}(x)}{r_{2 n}(-1)}=\cos \frac{\pi}{4}(x+1), \quad \lim _{n \rightarrow \infty} \frac{r_{2 n+1}(x)}{r_{2 n+1}(1)}=\sin \frac{\pi}{4}(x+1)$,

which hold uniformly throughout any finite domain. For our present purpose we shall need only an estimate for $\left|r_{n}(x)\right|$. Formula (29) is equivalent to

$$
r_{n}(x)=(-1)^{[n / 2]}\left(\frac{4}{\pi}\right)^{n+1} 2^{-3 / 2}\left(\cos \frac{\pi x}{4}+(-1)^{n-1} \sin \frac{\pi x}{4}\right)+r_{n}(x ; 1) ;
$$

as both $|\cos (\pi x / 4)|$ and $|\sin (\pi x / 4)|$ are $\leqq \cosh (\pi|x| / 4)$, it follows that

$$
\left|r_{n}(x)\right|<\left(\frac{4}{\pi}\right)^{n+1} 2^{-1 / 2} \cosh \frac{\pi|x|}{4}+K e^{|x|},
$$

where

$$
K=\frac{1}{2 \pi} \int_{|t|=1}\left|e^{t} \operatorname{sech} 2 t\right| \cdot|d t| .
$$

8. From (32) an estimate for $q_{n}(x)$, defined by (21), may readily be found. For let

$$
\gamma_{n}=n !\left|r_{n}(0)\right| ;
$$

(25). shows that the sign of $r_{n}(0)$ is $(-1)^{[n / 2]}$, hence the general formula (24) gives 


$$
\begin{aligned}
& r_{n}(x)= \\
& \frac{1}{n !}\left\{x^{n}+\left(\begin{array}{l}
n \\
1
\end{array}\right) \gamma_{1} x^{n-1}-\left(\begin{array}{l}
n \\
2
\end{array}\right) \gamma_{2} x^{n-2}-\left(\begin{array}{l}
n \\
3
\end{array}\right) \gamma_{3} x^{n-3}+\cdots+(-1)^{[n / 2]} \gamma_{n}\right\} .
\end{aligned}
$$

Substituting here $|x| / i$ for $x$, we get

$$
\begin{aligned}
& r_{n}\left(|x| i^{-1}\right) \\
& \quad=\frac{i^{-n}}{n !}\left\{|x|^{n}+i\left(\begin{array}{l}
n \\
1
\end{array}\right) \gamma_{1}|x|^{n-1}+\left(\begin{array}{l}
n \\
2
\end{array}\right) \gamma_{2}|x|^{n-2}+i\left(\begin{array}{l}
n \\
3
\end{array}\right) \gamma_{3}|x|^{n-3}+\cdots \cdot\right\},
\end{aligned}
$$

whence, by the inequality $|a|+|b| \leqq 2^{1 / 2}|a+i b|$ for $a$ and $b$ real,

$$
\begin{aligned}
\frac{1}{n !}\left\{|x|^{n}+\left(\begin{array}{l}
n \\
1
\end{array}\right) \gamma_{1}|x|^{n-1}+\left(\begin{array}{l}
n \\
2
\end{array}\right) \gamma_{2}|x|^{n-2}+\right. & \left.\cdots+\gamma_{n}\right\} \\
& \leqq 2^{1 / 2}\left|r_{n}\left(|x| i^{-1}\right)\right| .
\end{aligned}
$$

By Lemma 1 we have

$$
n !\left|q_{n}(0)\right|=\left|a_{n}\right| \leqq n !\left|r_{n}(0)\right|=\gamma_{n},
$$

and now (24) and (34) give

$$
\left|q_{n}(x)\right| \leqq 2^{1 / 2}\left|r_{n}\left(|x| i^{-1}\right)\right|
$$

This inequality and (32) give an estimate for $q_{n}(x)$. As the order of the lower limits of integration $\eta_{\nu}\left(-1 \leqq \eta_{\nu} \leqq 1\right)$ in $(21)$ is now immaterial, since the result holds for arbitrary $\eta_{\nu}$, their order may now be reversed. This proves

LEMMa 2. If

then

$$
\begin{aligned}
& p_{n}(x)=\int_{\eta_{0}}^{x} d x_{1} \int_{\eta_{1}}^{x_{1}} d x_{2} \cdots \int_{\eta_{n-1}}^{x_{n-1}} d x_{n} \\
&\left(-1 \leqq \eta_{\nu} \leqq 1, \nu=0,1, \cdots, n-1\right),
\end{aligned}
$$

$$
\left|p_{n}(x)\right|<\left(\frac{4}{\pi}\right)^{n+1} \cosh \frac{\pi|x|}{4}+2^{1 / 2} K e^{|x|}, *
$$

where $K$ is the numerical constant defined by (33).

* Goncharoff's general estimate

$$
\left|p_{n}(x)\right| \leqq\left(\left|x-\eta_{0}\right|+\left|\eta_{0}-\eta_{1}\right|+\cdots+\left|\eta_{n-2}-\eta_{n-1}\right|\right)^{n} / n !,
$$

which holds for arbitrary values of all quantities involved, is very favorable if $\eta_{n}$ tends to a limiting value for $n \rightarrow \infty$; it is less favorable, however, if, for example, $\eta_{n}=(-1)^{n+1}$. To establish his results mentioned in the introduction, Whittaker used the estimate

$\left(36^{\prime \prime}\right)$

$$
\left|p_{n}(x)\right| \leqq \frac{1}{2} e^{2}(1+|x|)^{n} \text { for }|x|>1,\left(\eta_{\nu}= \pm 1\right) .
$$

This estimate leads only to a finite region of convergence of (1), for functions $f(x)$ with the property (3), because the right side of $\left(36^{\prime \prime}\right)$ is very large for $n \rightarrow \infty$, if $|x|$ is large, whereas (36) permits to show that (1) converges in the whole plane if $f(x)$ satisfies (6) and $-1 \leqq \eta_{\nu} \leqq 1(\S 3)$. 
III. Convergence of the Abel Series expansion (1) FOR $-1 \leqq \eta_{\nu} \leqq 1$

9. Theorem 1 can now easily be proved. We need the following simple and well known lemma.

LEMMA 3. Let $f(x)$ be an integral function for which

$$
\varlimsup_{r \rightarrow \infty} \frac{\log M(r)}{r}=k<\infty \text {. }
$$

Then, if $l>k$,

$$
\left|f^{(n)}(0)\right|<l^{n} \text { for } n \geqq n_{0}(l) .
$$

Take $l^{\prime}$ such that $k<l^{\prime}<l$. By Cauchy's inequality

$$
\left|f^{(n)}(0)\right| \leqq \frac{M(r) n !}{r^{n}}<\frac{e^{l^{r} r} n !}{r^{n}} \quad\left(r \geqq r_{0}\right) .
$$

Taking $r=n / l^{\prime}$, this gives

$$
\left|f^{(n)}(0)\right|<\frac{e^{n} n !}{n^{n}} l^{\prime n}=\frac{e^{n} n !}{n^{n}}\left(\frac{l^{\prime}}{l}\right)^{n} \cdot l^{n}<l^{n} \quad\left(n \geqq n_{0}\right) .
$$

If $f(x)$ is an integral function we have, working formally with the expansion (1),

$$
\begin{aligned}
\sum_{n=0}^{\infty} f^{(n)}\left(\eta_{n}\right) p_{n}(x) & =\sum_{n=0}^{\infty} \sum_{r=0}^{\infty} \frac{f^{(n+r)}(0)}{r !} \eta_{n}^{r} p_{n}(x)=\sum_{m=0}^{\infty} f^{(m)}(0) \sum_{n+r=m} \frac{\eta_{n}^{r}}{r !} p_{n}(x) \\
& =\sum_{m=0}^{\infty} f^{(m)}(0) \frac{x^{m}}{m !}=f(x),
\end{aligned}
$$

for

$$
x^{m}=\eta_{0}{ }^{m}+m \eta_{1}{ }^{m-1} p_{1}(x)+\cdots+m ! p_{m}(x)=m ! \sum_{n+r=m} \frac{\eta_{n}^{r}}{r !} p_{n}(x),
$$

as a result of (1), if applied to $f(x)=x^{m}$. The formal relations (39) are certainly justified for $\left|\eta_{\nu}\right| \leqq 1$, if the double series

$$
\sum_{n=0}^{\infty} \sum_{r=0}^{\infty} \frac{1}{r !}\left|f^{(n+r)}(0)\right| \cdot\left|p_{n}(x)\right|
$$

is convergent. Let $f(x)$ satisfy (37), then (38) holds for $l>k$. On the other hand (36) gives

$$
\left|p_{n}(x)\right|<\left(\frac{4}{\pi}\right)^{n} A(\rho)+B(\rho) \text { for }|x| \leqq \rho .
$$


Hence (40) is dominated for $|x| \leqq \rho$ by the series

$$
\begin{aligned}
\sum_{n=0}^{\infty} \sum_{r=0}^{\infty} \frac{1}{r !} H l^{n+r}\left\{\left(\frac{4}{\pi}\right)^{n} A(\rho)\right. & +B(\rho)\} \\
= & \sum_{n=0}^{\infty} H e^{l l^{n}}\left\{\left(\frac{4}{\pi}\right)^{n} A(\rho)+B(\rho)\right\},
\end{aligned}
$$

$H$ being a suitable constant. If $k<\pi / 4$, we can choose $l$ such that $k<l<\pi / 4$, hence $4 l / \pi<1$ and also $l<1$. Then both series (41) and (40) converge and the expansion (1) is valid and absolutely and uniformly convergent in $|x| \leqq \rho$ for $\rho$ arbitrarily large.

Added in proof, June, 1936. The question whether or not the conditions $-1 \leqq \eta_{\nu} \leqq 1$ of Theorem 1 may be replaced by $\left|\eta_{\nu}\right| \leqq 1$ is still open. A general theorem of S. Takenaka, ${ }^{*}$ if suitably specialized, insures the validity of (1) around the origin under the assumptions $\left|\eta_{\nu}\right| \leqq 1$, provided $\gamma<\log 2$. With the method of the present note the matter hinges upon an extension of Lemma 2 to the case $\left|\eta_{\nu}\right| \leqq 1$. The estimate (36) may well hold under these more general assumptions.

* On the expansion of integral transcendental functions in generalized Taylor's series, Proceedings of the Physico-Mathematical Society of Japan, (3), vol. 14 (1932), pp. 529-542; see also the recent Cambridge Tract of J. M. Whittaker, Interpolatory Function Theory, Cambridge, 1935, where the same problem is pointed out as unsolved on p. 45 .

SWARThMore College,

SWARThMore, Pa. 